\title{
Influence of Filler Composition on the Refractive Index of Four Different Enamel Shades of Composite Resins
}

\author{
Rafael S Beolchi ${ }^{1}$, Deepak Mehta², Bruno Pelissier ${ }^{3}$, Luis A Gênova ${ }^{4}$, Anderson Z Freitas ${ }^{5}$, Shilpa H Bhandi ${ }^{6}$
}

\begin{abstract}
Aim and Objective: The purpose of this study was to measure the refractive index of four commercially available enamel resin composites, using optical coherence tomography (OCT) and their relationship with the atomic composition of the composite resin fillers utilizing an energydispersive X-ray fluorescence spectrometer (EDX).

Materials and methods: Four different enamel composites, namely Enamel HRi color UE3, Enamel HFO color GE3, Vit-I-escence color Pearl Frost, and Amelogen Plus color Enamel White were tested. For each composite, disks with different thicknesses were fabricated and then lightcured according to the manufacturer's instructions. The disks were then stored in deionized water under $36.5^{\circ} \mathrm{C}$ for at least 7 days. Each of the samples from the four different groups was measured in five distinctive points to analyze the optical and physical length using optical coherence tomography (OCT). Elemental analysis of all four different enamel shades of the composite was done using an EDX.

Results: The filler contents showed interesting differences in elemental composition and concentration; however, Si seemed to be a common filler component. The HRi composite presented a distinctive composition compared to other materials and was the only composite that showed a smaller percentage of $\mathrm{SiO}_{2}$, and also was the only composite that contained compounds, such as $\mathrm{P}_{2} \mathrm{O}_{5}, \mathrm{ZnO}, \mathrm{CaO}, \mathrm{La}_{2} \mathrm{O}_{3}$, and V $\mathrm{O}_{5}$. The optical coherence tomography analysis showed the refractive index values of all tested enamel composites. Among the four different enamel composite resins tested, the enamel HRi composite demonstrated the most ideal refractive index to mimic natural enamel.

Conclusion: The enamel HRi composite demonstrated a distinctive filler composition and this could be the main reason behind its higher refractive index. Nonetheless, it remains unclear how much of an impact this feature has in the final esthetic outcome of anterior composite restorations, where many other optical phenomena are also important.

Clinical significance: The clinical success of any esthetic restorative procedure depends on diagnosing the proper treatment plan and also on executing this treatment with the right materials. On direct esthetic restorations, knowing the optical properties of such materials is fundamental, as they should be able to replicate both natural enamel and dentin.

Keywords: Dental composites, Energy-dispersive X-ray fluorescence spectrometer, Filler composition, Optical coherence tomography, Refractive index.

The Journal of Contemporary Dental Practice (2021): 10.5005/jp-journals-10024-3040
\end{abstract}

\section{INTRODUCTION}

Since the introduction of composite resins to the dental profession by $\mathrm{Dr}$. Bowen in the 1960s, composite resins have proven to be predictable and reliable restorative materials for the treatment of traumatized dentin or dental caries in both the anterior and posterior regions. ${ }^{1,2}$ Currently, resin composites are undisputedly one of the most widely used materials in restorative dentistry. 3,4 Greater aesthetic demand from the patients contributed to an increase in aesthetic restorations over the past few years. Resultantly, a new stage in modern adhesive aesthetic dentistry has begun.

The mechanical properties, especially the optical properties of resin composites have been affected by variations in filler size, shape, and composition. ${ }^{5}$ Therefore, the relationship between aesthetic properties and different components of the composite fillers currently used in dentistry provides important information to both clinicians and researchers. Today, many modern dental composites present optical characteristics that resemble those of natural dentition. Materials with different opacities, different colors, and also more subtle optical properties, such as fluorescence and opalescence are now available for clinicians.

\footnotetext{
'Department of Dentistry, São Leopoldo Mandic, Campinas, São Paulo, Brazil

${ }^{2}$ Department of Preventive and Restorative Dentistry, College of Dental Medicine, University of Sharjah, Sharjah, UAE

${ }^{3}$ Department of Restorative Dentistry, UFR d'Odontologie, Montpellier, France

${ }^{4}$ Materials Science and Technology Center, Nuclear and Energy Research Institute, São Paulo, Brazil

${ }^{5}$ Applied Laser Center, Nuclear and Energy Research Institute, São Paulo, Brazil

${ }^{6}$ Department of Restorative Dental Science, Division of Operative Dentistry, College of Dentistry, Jazan University, Jazan, Kingdom of Saudi Arabia
}

Corresponding Author: Deepak Mehta, Department of Preventive and Restorative Dentistry, College of Dental Medicine, University of Sharjah, Sharjah, UAE, Phone: +919845140082, e-mail: dmehta@sharjah.ac.ae

How to cite this article: Beolchi RS, Mehta D, Pelissier B, et al. Influence of Filler Composition on the Refractive Index of Four Different Enamel Shades of Composite Resins. J Contemp Dent Pract 2021;22(5):557-561.

Source of support: Nil

Conflict of interest: None

() Jaypee Brothers Medical Publishers. 2021 Open Access This article is distributed under the terms of the Creative Commons Attribution 4.0 International License (https://creativecommons.org/licenses/by-nc/4.0/), which permits unrestricted use, distribution, and non-commercial reproduction in any medium, provided you give appropriate credit to the original author(s) and the source, provide a link to the Creative Commons license, and indicate if changes were made. The Creative Commons Public Domain Dedication waiver (http://creativecommons.org/publicdomain/zero/1.0/) applies to the data made available in this article, unless otherwise stated. 
Similarly, the use of dental composites for enamel with the closest match of refractive index to natural enamel would hopefully present an ideal outcome of the optical characteristics found in nature. ${ }^{6}$ This could be one reason why aesthetic problems may arise when restoring the enamel on anterior teeth, due to the refractive index mismatch of the natural versus the artificial material. Clinically, such mismatch may cause a shadow-like effect on the margins between the teeth and the restoration due to the destructive interference phenomenon of the light when it travels within two materials with distinct refractive indexes. ${ }^{7}$ This phenomenon is similar to thin-film interference. ${ }^{8}$

Presently, a relatively successful method for measuring a tooth's refractive index is the OCT method. ${ }^{9-11}$ Optical coherence tomography (OCT), a novel biological imaging technology, has been developed rapidly over the last decades. One of the applications of the OCT is the assessment of the refractive indexes of non-clear materials, such as human enamel and dental composites. $^{12}$

Considering this problem, nowadays there are dental composite materials that claim to have the same refractive index as the natural enamel. The null hypothesis assumed in the present study was that the filler composition did not influence the refractive indices of different enamel shades of resin composites. The objective of this study was to measure the refractive indices of four commercially available dental composites by using OCT and correlate the resulting data with the analysis of the atomic composition of the composite fillers using an energy-dispersive X-ray fluorescence spectrometer (EDX).

\section{Materials and Methods}

The present study was conducted at the Laser Applications Center (CLA), Nuclear and Energy Research Institute (IPEN), University of Sao Paulo (USP), Brazil.

\section{Enamel Resin Composites and Curing Light}

Four enamel composites were tested in this study: Enamel HRi color UE3 (Micerium S.p.A., Italy), Enamel HFO color GE3 (Micerium S.p.A., Italy), Vit-I-escence color Pearl Frost (Ultradent Products Inc., USA), and Amelogen Plus color Enamel White (Ultradent Products Inc., USA). Three resin composite disks, each $5 \mathrm{~mm}$ in diameter, were tested from four different enamel composites.

These four brands were selected since they reproduce natural enamel and are fabricated following the non-vita enamel layering technique. In other words, they follow the clinical pattern of achromatic, colorless, and enamel colors. The colors selected were those required for the reproduction of high-value enamel.

Each composite disk of $5 \mathrm{~mm}$ in diameter, with different thicknesses ranging from 0.15 to $0.44 \mathrm{~mm}$ (Table 1) was fabricated and then light-cured according to the manufacturer's instructions. All disks were light-cured for 20 seconds, using a Valo curing light (Ultradent Products Inc., USA). This device allows for three different intensities, and the light intensity (exitance) used was that of $1000 \mathrm{~mW} / \mathrm{cm}^{2}$, with a wavelength in the range of $395-480 \mathrm{~nm}$. The total amount of energy delivered was $20 \mathrm{~J} / \mathrm{cm}^{2}$. The disks were then stored in deionized water under $36.5^{\circ} \mathrm{C}$ for at least 7 days.

\section{Optical Coherence Tomography (OCT)}

The OCT experiment was conducted with a device for optical coherence tomography (ThorLabs, Lübeck, Germany), with a wavelength of $930 \mathrm{~nm}$. The equipment is integrated with software for image generation and an XYZ translator. For the image analyses, the free software ImageJ was used.

The four kinds of abovementioned composites were tested, with three different sample thicknesses for each one. Each of the samples was measured in five distinctive points, to assess the material homogeneity and to make the measurements more trustful.

Composite samples were placed over a flat glass surface, and then both were placed over the translator, enabling the sample to be moved both horizontally and vertically.

The resulting images were then analyzed for both the optical and physical lengths, through the following equation: $n=o l / p l$, where $\mathrm{n}$ is the refractive index, ol is the optical length, and $\mathrm{pl}$ is the physical length (Fig. 1).

\section{Energy-dispersive X-ray Fluorescence (EDX)}

Elemental analysis of the enamel-based resin composites was performed using an EDX. The specimens were fabricated using a proper matrix for the EDX spectrometer, in a thickness of $2 \mathrm{~mm}$, and were light-cured in the same way as described above for the OCT technique. The specimens were then placed on the EDX spectrometer stage without any coating. The analysis was then performed at the center of each specimen.

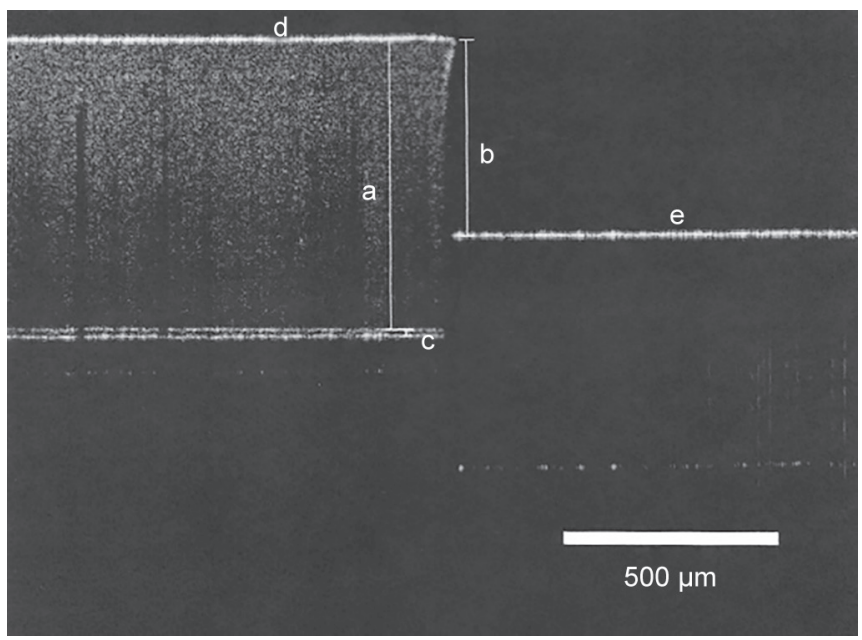

Fig. 1: OCT image of Enamel HRi resin composite. The "a" segment represents the optical length; the " $b$ " segment represents the physical length; "c" is the air cushion under the composite; " $d$ " is the composite's top; and " $\mathrm{e}$ " is the glass plate surface on which the composite was resting

Table 1: Composite sample thickness and refractive index according to OCT measurement

\begin{tabular}{|c|c|c|c|c|c|c|c|c|c|c|c|c|}
\hline Composite & Enamel & & & Enamel & HFO & & Amelog & en Plus & & Vit-l-esc & ence & \\
\hline Real thickness $(\mu \mathrm{m})$ & 155.82 & 312.25 & 440.97 & 160.04 & 322.59 & 442.37 & 15411 & 305.47 & 450.30 & 157.73 & 331.53 & 447.26 \\
\hline Approx. thickness (mm) & 0.15 & 0.31 & 0.44 & 0.16 & 0.32 & 0.44 & 0.15 & 0.30 & 0.45 & 0.16 & 0.33 & 0.44 \\
\hline RI & 1.59 & 1.59 & 1.59 & 1.55 & 1.51 & 1.53 & 1.56 & 1.58 & 1.55 & 1.54 & 1.52 & 1.52 \\
\hline
\end{tabular}




\section{ResUlts}

\section{Refractive Index}

Table 1 presents the values of optical length vs physical length and also the resulting refractive index (RI) for each composite sample.

\section{Chemical Composition}

The chemical compositions of the different enamel composite resins including the elements with relative values expressed in weight percentage are presented in Table 2.

The filler contents show significant differences in elemental composition and concentration; nevertheless, Si seems to be a common filler component, as it was $\mathrm{CH}$, related to the carbonic composition of the organic, resinous matrix of all composites.

The Enamel HRi demonstrated consistent refractive index values similar to human enamel measured at varying thicknesses. At different thicknesses of $0.15,0.31$, and $0.44 \mathrm{~mm}$, the refractive index value of Enamel HRi remained consistent at 1.59. The Enamel HFO refractive index value ranged from 1.51 to 1.55 with varying thicknesses of $0.16,0.32$, and $0.44 \mathrm{~mm}$.

Amelogen Plus was measured at $0.15,0.30$, and $0.45 \mathrm{~mm}$ thickness and the refractive index values ranged between 1.55 and 1.58. The Vit-I-escence enamel composites showed refractive index values of 1.52 and 1.54 at thickness values of $0.16,0.33$, and $0.44 \mathrm{~mm}$. However, the Enamel HFO, Amelogen Plus, and Vit-Iescence showed inconsistent refractive index values with varying thickness of composite resin when compared to Enamel HRi which was consistent at the different thickness.

\section{Discussion}

The null hypothesis was rejected because as it seems, the filler composition influenced the refractive index of different enamel shades of resin composites. The addition of filler particles has been the most critical factor in the development of a newer generation of composite resins, since the scattering and absorption can be controlled for the best color reproduction, considering different refractive indices of filler and resin matrix. ${ }^{13}$

To outline the restorations against dentin and enamel, radiopaque additives (e.g., barium doped glass) are added to

Table 2: Atomic composition of each composite, in \% per atomic mass

\begin{tabular}{lcccc}
\hline & $H R I$ & $H F O$ & $V I T$ & APLUS \\
\hline $\mathrm{SiO}_{2}$ & 27.751 & 53.032 & 52.311 & 35.203 \\
$\mathrm{P}_{2} \mathrm{O}_{5}$ & 19.167 & & & \\
$\mathrm{ZrO}_{2}$ & 8.208 & & & \\
$\mathrm{SrO}$ & 7.096 & 7.79 & 7.636 & 0.088 \\
$\mathrm{ZnO}$ & 3.375 & & & \\
$\mathrm{Al}_{2} \mathrm{O}_{3}$ & 3.04 & 9.824 & 9.393 & 20.643 \\
$\mathrm{CaO}$ & 2.44 & & 0.055 & \\
$\mathrm{La}_{2} \mathrm{O}_{3}$ & 1.084 & & & \\
$\mathrm{~V}_{2} \mathrm{O}_{5}$ & 0.182 & & & \\
$\mathrm{HfO}_{2}$ & 0.179 & & & \\
$\mathrm{CH}$ & 27.478 & 28.55 & 29.743 & 21.421 \\
$\mathrm{BaO}_{\mathrm{SO}}$ & & 0.0803 & 0.862 & 22.542 \\
$\mathrm{CdO}$ & & & & 0.072 \\
$\mathrm{CuO}$ & & & & 0.019 \\
\hline
\end{tabular}

increase the radiopacity of composite resins. ${ }^{14}$ Sometimes, additional additives are also used, like $\mathrm{Sr}, \mathrm{Zr}, \mathrm{Yb}$, and La. Besides possessing radiopacity, these bigger atoms are also fluorescent. This feature helps to increase the optical properties of the composites compared to the natural tooth. The natural fluorescence of the tooth, according to Fukushima et al. $^{15}$ is due to the presence of piridinoline, a collagen amino acid responsible for its cross-linking. Other studies ${ }^{16}$ have attested that besides piridinoline, which fluoresces when excited under $<325 \mathrm{~nm}$ and emits in the range of $300 \mathrm{~nm}$, three more elements might be responsible for the phenomena: tryptophan (excitation at $300 \mathrm{~nm}$ and emission at $350 \mathrm{~nm}$ ) and two still unknown chromophores, which excites at 380 and $410 \mathrm{~nm}$ and emits at 450 and $520 \mathrm{~nm}$ respectively.

However, since the abovementioned atomic elements have higher atomic numbers, the translucency under visible light of the resin composites is restricted. The silane coupling agent, responsible for augmenting the bond between the filler and matrix, could also cause a decrease in translucency. Assimilating a greater percentage of radiopaque fillers leads to incongruity in the refractive index between the filler and the resin matrix. ${ }^{12}$ Watts and Cash $^{17}$ demonstrated that the material's composition is a determining factor of its optical properties. The EDX spectrometer is an instrument that quantitatively determines the elements within a sample by irradiating the sample with X-rays and then analyzing the re-emitted fluorescent X-rays. The HRi enamel composite evaluated presented a distinctive composition compared to the other materials; it was the only composite that showed a smaller percentage of $\mathrm{SiO}_{2}$, and also was the only material that contained materials, such as $\mathrm{P}_{2} \mathrm{O}_{5}, \mathrm{ZnO}, \mathrm{CaO}, \mathrm{La}_{2} \mathrm{O}_{3}$, and $\mathrm{V}_{2} \mathrm{O}_{5}$. The presence of $\mathrm{HfO}_{2}$ in such a small concentration can be explained by the fact that this is a common finding when in the presence of $\mathrm{Si}$, as both elements present similar EDX values, and those may sometimes overlap.

Other elements found in all composites were $\mathrm{SrO}$ and $\mathrm{Al}_{2} \mathrm{O}_{3}$. The Amelogen Plus composite also showed traces of $\mathrm{SO}_{3}, \mathrm{CdO}$, and $\mathrm{CuO}$. A common element found in several composites is the $\mathrm{Fe}_{2} \mathrm{O}_{3}$, which is used as an opaque brownish pigment, usually as dentin colors, but it was not found in our study since the materials tested are composites meant to reproduce natural colorless translucent enamel. Filler composition, content, shape, and size are some of the factors responsible for the optical dispersion of a material, and for the light transmittance characteristics of composite resins.

In recent years, the refractive index mismatch has been addressed because clinically it may cause a shadow-like effect around the margins of the restoration due to the destructive interference phenomena of the light when it travels through two materials with different refractive indexes. If that mismatch is enough to make light waves having equal wavelength occupy the same physical space, they will interfere with each other in a manner determined by their relative phase and amplitude. The interference is destructive if the waves are exactly out of phase (by 180 degrees), and if their amplitudes are equal, they cancel each other out to yield a wave of zero amplitude.

The refractive index or the relationship between the speed of the light in a vacuum and a concrete body is a distinctive property of matter typically used for identifying materials., ${ }^{6,9}$ When the refractive index of light in the vacuum coincides with the index in the medium, without path modification, the medium is called transparent. If the refractive indices are different, the medium would present distinct translucent or opaque characteristics. ${ }^{5}$ 
It is known that natural human enamel has a refractive index ranging around $1.62 \pm 0.02 .{ }^{5}$ Recently, another study refined these data using OCT, providing more specific values, $1.631 \pm 0.007$. $^{5}$ Complete transference of light by enamel increased with increasing wavelength. Tooth enamel is more translucent at higher wavelengths, ${ }^{18}$ which shows the bi-refringence characteristic of this tissue, where the prisms are the most important scatters, but the crystals are responsible for the back-scattering. ${ }^{19}$

Some of the composites meant to reproduce enamel have a refractive index of $1.52 .^{2}$ Our present study provides specific results for each brand, and the values ranged from 1.51 to 1.59 .

The OCT setup is a Michelson's interferometer with a light source, a beam splitter, a reference mirror, a detector, and the sample itself. The beam splitter divides the light emitted by the source. Then, approximately half of its intensity is directed to the mirror and the other half is directed to the sample. The light emitted by the sample and the light reflected by the mirror are recombined at the beam splitter and interference patterns are identified by the detector. Because of the broadband property of the light source, the interference pattern will occur only when the optical paths difference between these two arms (reference and sample) are the same. This property allows the control of the depth of light penetration into the sample. Because of this property, it is possible to use OCT to assess the refractive indexes, since the measurements made by OCT are optical lengths and not physical lengths.

By analyzing the images, it was possible to determine that the size of the composites is its optical size, and that length between its surface and the glass plate in which the composites were standing corresponds to its physical size, because light traveled through the air, and not inside the composite. Another important feature is that the measurement was made using an infra-red laser at $930 \mathrm{~nm}$ in the non-visible spectrum. It is not possible to correlate the values obtained in this non-visible wavelength to those that can be attained using a smaller, visible wavelength. ${ }^{20}$

It can be anticipated that the use of enamel shades of composites having the same refractive index as tooth enamel would account for a complete reproduction of the visual characteristics established in nature. Unfortunately, having the right refractive index is only one of the many ingredients imperative for providing realistic restorations. Selecting artificial dentin and enamel shades of proper opacities, and regulating the accurate thickness of each layer, are of prime consequence and should be just as important (or even more) as the use of refractive indices. ${ }^{2}$

The present study highlights the importance of filler composition and its role in light transmission. However, it remains unclear to determine the extent of the impact of this feature on the aesthetic outcome of anterior composite restorations, where many other optical phenomena are also important.

More research is needed towards evaluating the refractive index of different materials for resin composites. However, further variables are required to be measured to analyze the behavior of light and its propagation. Also, the role of composite thickness seems to be the foremost factor considering the refractive index. Future studies are needed to evaluate the role of different thicknesses, filler composition, and light transmission.

\section{Conclusion}

Within the limitations of this in vitro experimental study, varying inorganic filler composition characteristics among the evaluated enamel composite resins were found.
However, it may be inferred that the Enamel HRi composite had the closest refractive index to human enamel when compared to other enamel shades investigated.

An understanding of the shade and optical properties of resin composites is integral to obtain lifelike restorations. The clinical success of these restorative procedures depends on selecting appropriate materials that replicate the natural enamel and provide long-term color stability.

\section{References}

1. Jaarda MJ, Lang BR, Wang RF, et al. Measurement of composite resin filler particles by using scanning electron microscopy and digital imaging. J Prosthet Dent1993;69(4):416-424. DOI: 10.1016/00223913(93)90191-P.

2. Zandinejad AA, Atai M, Pahlevan A. The effect of ceramic and porous fillers on the mechanical properties of experimental dental composites. Dent Mater 2006;22(4):382-387. DOI: 10.1016/j. dental.2005.04.027.

3. da Silva GR, Simamto-Junior PC, da Mota AS, et al. Mechanical properties of light-curing composites polymerized with different laboratory photo-curing units. Dent Mater 2007;26(2):217-223. DOI: 10.4012/dmj.26.217.

4. Wilson NH, Dunne SM, Gainford ID. Current materials and techniques for direct restorations in posterior teeth. Part 2: Resin composites systems. Int Dent J 1997;47(4):185-193. DOI: 10.1111/j.1875-595X.1997. tb00448.x.

5. Asaka Y,Miyazaki M,AboshiH, etal.AEDXfluorescenceanalysis and SEM observations of resin composites. J Oral Science 2004;46(3):143-148. DOI: 10.2334/josnusd.46.143.

6. Wang XJ, Milner TE, de Boer JF, et al. Characterization of dentin and enamel by use of optical coherence tomography. Appl Opt 1999;38(10):2092-2096. DOI: 10.1364/AO.38.002092.

7. Villarroel M, FahI N, De Sousa AM, et al. Direct esthetic restorations based on translucency and opacity of composite resins. J Esthet Restor Dent 2011;23(2):73-87. DOI: 10.1111/j.17088240.2010.00392.x.

8. Schrenk WJ, Wheatley JA, Lewis RA, et al. Nanolayer polymeric optical films. TAPPI 1991 Polymers, laminations and coatings conference. Norcross, GA: TAPPI; 1991. pp. 233-240.

9. Meng $\mathrm{Z}$, Yao $\mathrm{H}$, Liang $\mathrm{Y}$, et al. Measurement of the refractive index of human teeth by optical coherence tomography. J Biomed Opt 2009;14(3):034010. DOI: 10.1117/1.3130322.

10. Haaruna m, Ohmi m, Mitsuyama t, et al. Simultaneous measurement of the phase and group indices and the thickness of transparent plates by low coherence interferometry. Opt Lett 1998;23(12):966968. DOI: 10.1364/OL.23.000966.

11. Tearney GJ, Brezinski M, Southern JF, et al. Determination of the refractive index of highly scattering human tissue by optical coherence tomography. Opt Lett 1995;120(21):2258-2260. DOI: 10.1364/OL.20.002258.

12. Freitas AZ, Amaral MM, Raele MP. Optical coherence tomography: development and applications. Laser Pulse Phenomena and Applications, D.F.J. Duarte, ed. Intech; 2010. pp. 409-432. DOI: $10.5772 / 12899$.

13. Yong-Keun L. Influence of filler on the difference between the transmitted and reflected colors of experimental resin composites. Dent Mater 2008;24(9):1243-1247. DOI: 10.1016/j. dental.2008.01.014.

14. Tarumi H, Torii M, Tsuchitani Y. Relationship between particle size of barium glass filler and water sorption of light cured composite resin. Dent Mater J 1995;14(1):37-44. DOI: 10.4012/dmj.14.37.

15. Fukushima Y, Araki T, Yamada MO. Topography of fluorescence and its possible composites in human teeth. Cell Mol Biol 1987;33(6):725-736.

16. Zijp JR. Optical properties of dental hard tissues. University of Groningen: s.n. 2001. 
17. Watts DC, Cash AJ. Analysis of optical transmission by $400-500 \mathrm{~nm}$ visible light into aesthetic dental biomaterials. J Dent 1994;22(2):112117. DOI: 10.1016/0300-5712(94)90014-0.

18. Baumgartner A, Dichtl S, Hitzenberger $C K$, et al. Polarization-sensitive optical coherence tomography of dental structures. Caries Res 2000;34(1):59-69. DOI: 10.1159/000016571.
19. Brodbelt $\mathrm{RH}, \mathrm{O}^{\prime}$ brien WJ, Fan PL, et al. Translucency of human dental enamel. J Dent Res 1981;60(10):1749-1753. DOI: 10.1177/00220345810600100401.

20. Zijp JR, ten bosch JJ, Groenhuis RAJ. HeNe-laser light scattering by human dental enamel. J Dent Res 1995;74(12):1891-1898. DOI: $10.1177 / 00220345950740121301$. 